\title{
CDSeq on deep deconvolution
}

A

\section{Correlation: estimated GEPs vs true GEPs}

\begin{tabular}{|l|l|l|l|l|l|l|l|l|l|l|l|l|l|l|l|l|l|l|l|l|l|l|}
\hline B cells naive & 0.23 & 0.21 & 0 & 0.17 & 0.14 & 0.21 & 0.12 & 0.18 & 0.14 & 0.14 & 0.11 & 0.12 & 0.07 & 0.03 & -0.08 & 0.04 & 0.07 & -0.05 & -0.02 & 0 & 0.1 & 0.1 \\
\hline
\end{tabular}

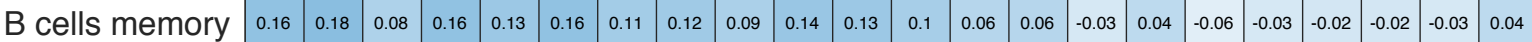

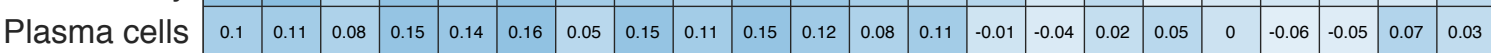

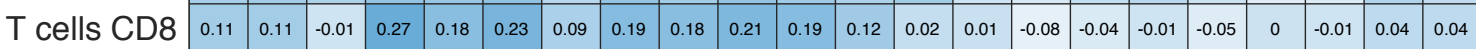

\begin{tabular}{|l|l|l|l|l|l|l|l|l|l|l|l|l|l|l|l|l|l|l|l|l|l|l|}
\hline T cells CD4 naive & 0.13 & 0.16 & 0.09 & 0.33 & 0.27 & 0.3 & 0.22 & 0.2 & 0.26 & 0.27 & 0.16 & 0.14 & 0.14 & 0.03 & 0.06 & 0.05 & 0.01 & 0.05 & -0.05 & -0.04 & 0.04 & 0.1 \\
\hline
\end{tabular}

\begin{tabular}{|l|l|l|l|l|l|l|l|l|l|l|l|l|l|l|l|l|l|l|l|l|l|l|}
\hline T cells CD4 memory resting & 0.1 & 0.09 & 0.06 & 0.26 & 0.21 & 0.25 & 0.16 & 0.21 & 0.2 & 0.16 & 0.07 & 0.05 & 0.1 & 0.06 & -0.06 & 0.01 & -0.02 & 0 & 0.02 & 0.03 & 0.05 & 0.11 \\
\hline
\end{tabular}

T cells follicular helpe

T cells regulatory (Tregs)

$\mathrm{T}$ cells gamma delta

NK cells resting

NK cells activated

Monocytes

Macrophages M0

Macrophages M1

Macrophages M2

Dendritic cells resting

Dendritic cells activated

Mast cells resting

Mast cells activated

Eosinophils

Neutrophils

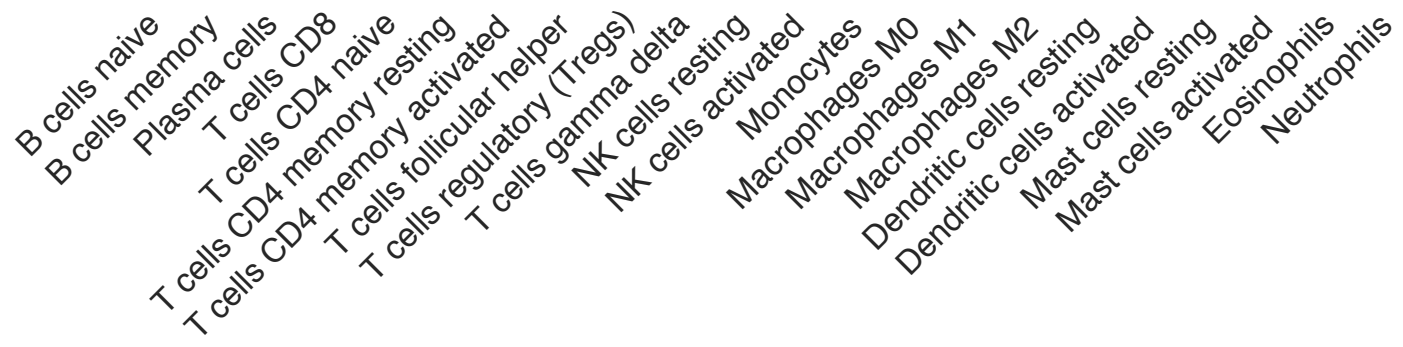

LM22 True GEPs 

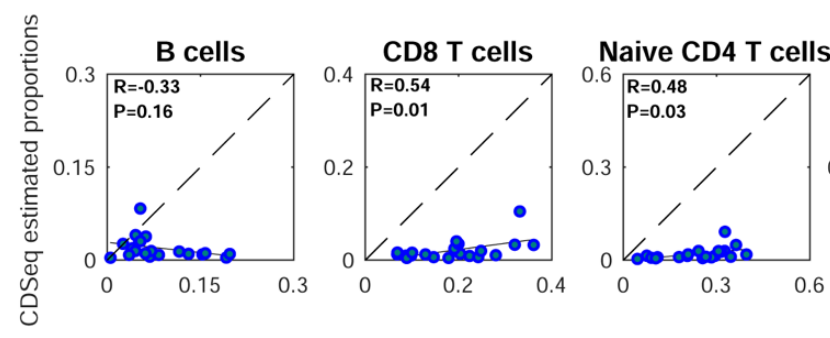

Resting memory Activated memory
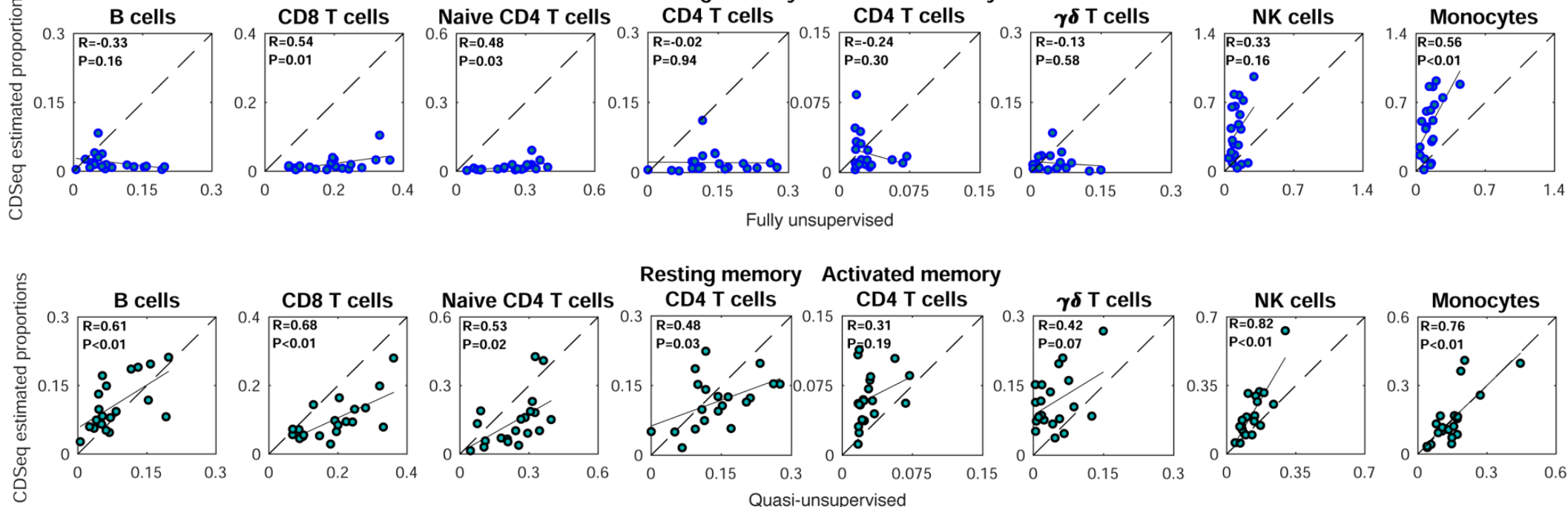

Resting memory Activated memory
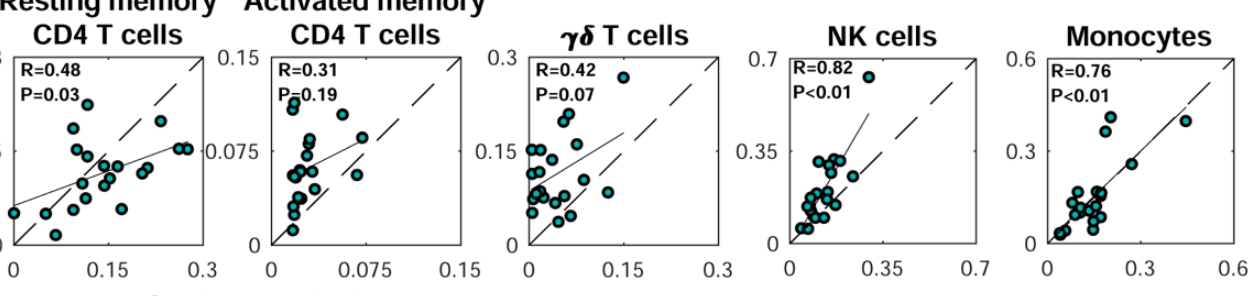

Quasi-unsupervised 


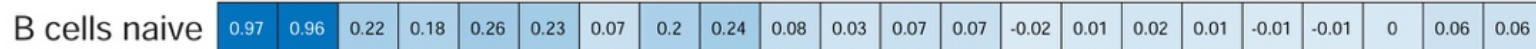

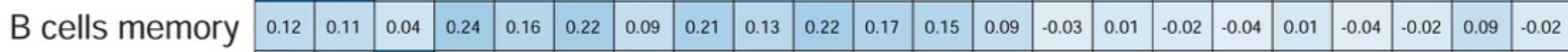

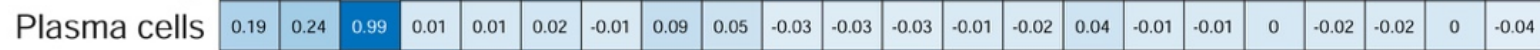

\begin{tabular}{l|l|l|l|l|l|l|l|l|l|l|l|l|l|l|l|l|l|l|l|l|l|l|} 
T cells CD8 & 0.17 & 0.19 & 0.01 & 0.99 & 0.72 & 0.82 & 0.36 & 0.57 & 0.54 & 0.75 & 0.55 & 0.44 & -0.02 & -0.02 & 0.01 & -0.07 & -0.06 & 0.04 & -0.03 & -0.02 & 0 & 0.01
\end{tabular}

\begin{tabular}{|l|l|l|l|l|l|l|l|l|l|l|l|l|l|l|l|l|l|l|l|l|l|l|l}
\hline T cells CD4 naive & 0.26 & 0.26 & 0.01 & 0.71 & 0.99 & 0.87 & 0.33 & 0.7 & 0.64 & 0.31 & 0.13 & 0.13 & 0 & 0.01 & 0 & -0.04 & -0.04 & 0.06 & -0.03 & -0.04 & 0.03 & 0.05 \\
\hline
\end{tabular}

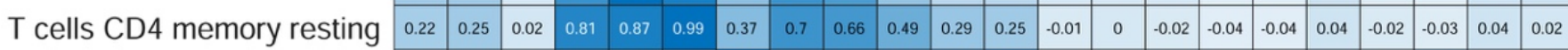

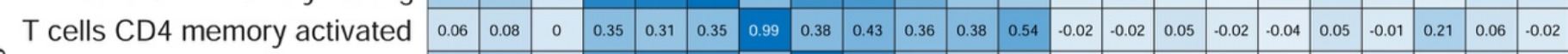

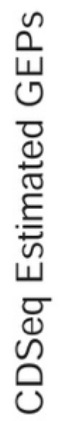

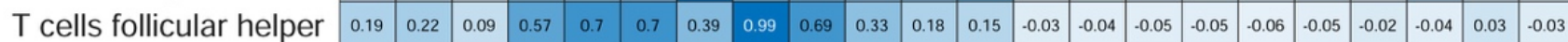

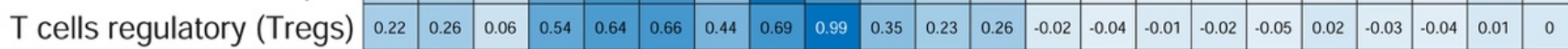

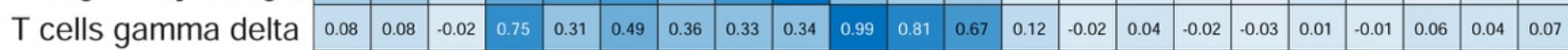

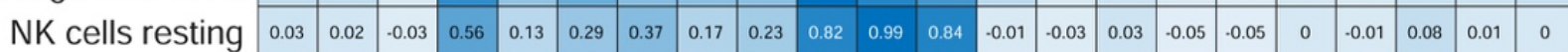

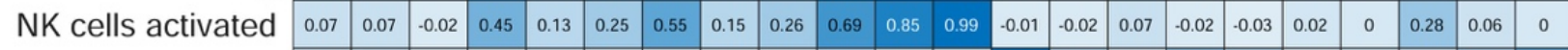

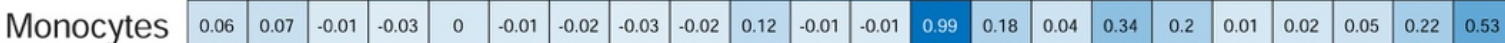

\begin{tabular}{|c|c|c|c|c|c|c|c|c|c|c|c|c|c|c|c|c|c|c|c|c|c|c|}
\hline Macrophages M0 & -0.02 & -0.01 & -0.01 & -0.02 & 0.01 & 0 & -0.02 & -0.04 & -0.04 & -0.02 & -0.03 & -0.02 & 0.18 & 0.99 & 0.06 & 0.56 & 0.33 & 0.16 & 0.01 & 0.18 & 0.15 & 0.25 \\
\hline
\end{tabular}

\begin{tabular}{|l|l|l|l|l|l|l|l|l|l|l|l|l|l|l|l|l|l|l|l|l|l|l|}
\hline Macrophages M1 & 0.01 & 0.01 & 0.05 & 0.01 & 0 & -0.02 & 0.06 & -0.05 & -0.01 & 0.04 & 0.03 & 0.07 & 0.04 & 0.06 & 0.99 & 0.08 & 0.02 & 0.54 & -0.02 & 0.07 & 0.05 & 0.03 \\
\hline
\end{tabular}

\begin{tabular}{|l|l|l|l|l|l|l|l|l|l|l|l|l|l|l|l|l|l|l|l|l|l|l|} 
Macrophages M2 & 0.01 & 0.03 & -0.01 & -0.07 & -0.04 & -0.04 & -0.02 & -0.05 & -0.02 & -0.01 & -0.05 & -0.02 & 0.34 & 0.56 & 0.08 & 0.99 & 0.42 & 0.12 & 0.02 & 0.13 & 0.15 & 0.19 \\
\hline
\end{tabular}

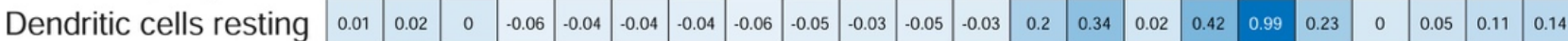

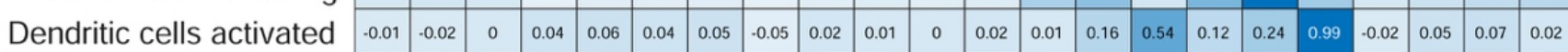

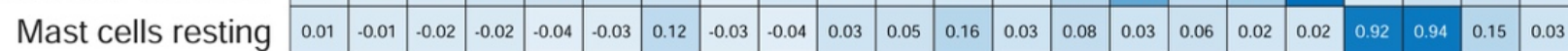

\begin{tabular}{|l|l|l|l|l|l|l|l|l|l|l|l|l|l|l|l|l|l|l|l|l|l|l|}
\hline Mast cells activated & 0.03 & 0.02 & 0.03 & 0.06 & 0.01 & 0.04 & 0.01 & 0.09 & 0 & 0.11 & 0.06 & 0.05 & 0.32 & 0 & -0.01 & 0.04 & -0.01 & 0.02 & -0.01 & 0.06 & 0.09 & 0.06 \\
\hline
\end{tabular}

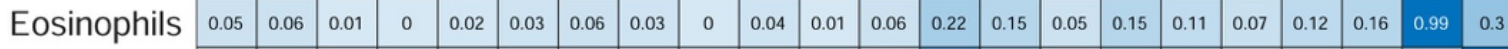

\begin{tabular}{|l|l|l|l|l|l|l|l|l|l|l|l|l|l|l|l|l|l|l|l|l|l|l|} 
Neutrophils & 0.05 & 0.06 & -0.04 & 0 & 0.05 & 0.02 & -0.02 & -0.03 & 0 & 0.07 & 0 & 0 & 0.54 & 0.25 & 0.03 & 0.19 & 0.14 & 0.02 & 0.03 & 0.06 & 0.3 & 0.99 \\
\cline { 2 - 9 }
\end{tabular}

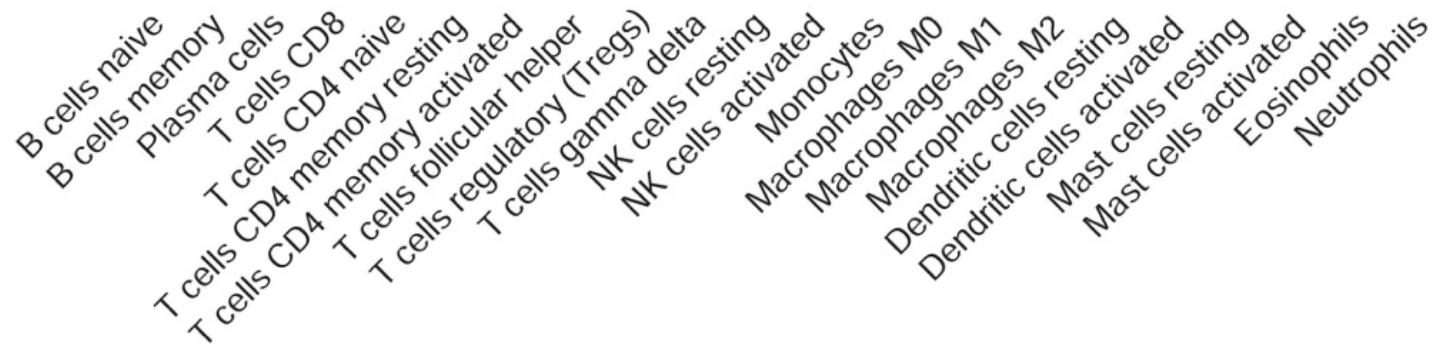

LM22 True GEPs 


\section{Correlation: estimated GEPs vs true GEPs}

\begin{tabular}{|l|l|l|l|l|l|l|l|l|l|l|l|l|l|l|l|l|l|l|l|l|l|l|}
\hline B cells naive & 0.18 & 0.14 & 0.03 & 0.15 & 0.13 & 0.12 & 0.1 & 0.08 & 0.16 & 0.05 & 0.04 & 0.03 & 0.03 & 0.01 & 0.1 & -0.02 & -0.04 & 0.17 & -0.03 & -0.01 & 0.01 & 0.03 \\
\hline
\end{tabular} \begin{tabular}{|l|l|l|l|l|l|l|l|l|l|l|l|l|l|l|l|l|l|l|l|l|l|l|l|}
\hline B cells memory & -0.15 & -0.15 & -0.01 & -0.21 & -0.18 & -0.22 & -0.13 & -0.19 & -0.18 & -0.2 & -0.16 & -0.12 & -0.19 & -0.1 & -0.04 & -0.09 & -0.04 & -0.04 & 0.01 & 0 & -0.05 & -0.16 \\
\cline { 2 - 8 }
\end{tabular}

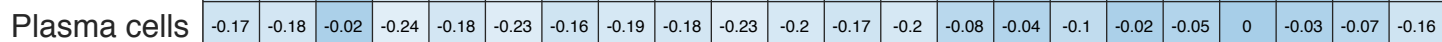

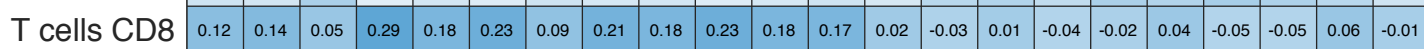

\begin{tabular}{|l|l|l|l|l|l|l|l|l|l|l|l|l|l|l|l|l|l|l|l|l|l|l|}
\hline T cells CD4 naive & 0.15 & 0.16 & 0.08 & 0.21 & 0.25 & 0.26 & 0.1 & 0.25 & 0.2 & 0.18 & 0.11 & 0.11 & 0.41 & 0.01 & 0 & 0.1 & 0.05 & 0.03 & -0.02 & -0.02 & 0.11 & 0.1 \\
\hline
\end{tabular}

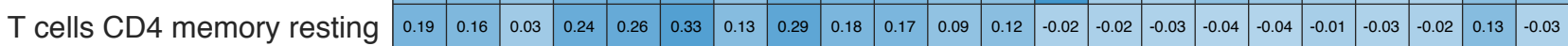
T cells CD4 memory activated

T cells follicular helper

$T$ cells regulatory (Tregs)

T cells gamma delta

NK cells resting

NK cells activated

Monocytes

Macrophages M0

Macrophages M1

Macrophages M2

Dendritic cells resting

Dendritic cells activated

Mast cells resting

Mast cells activated

Eosinophils

Neutrophils

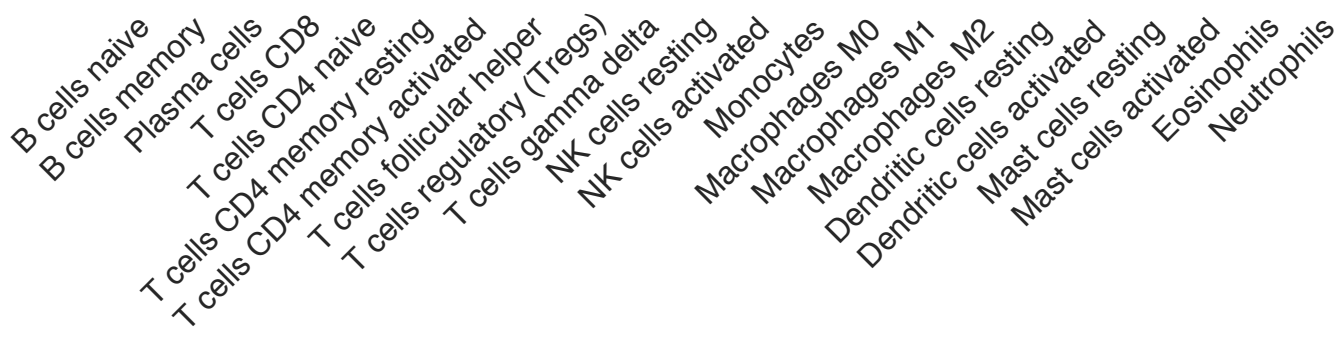

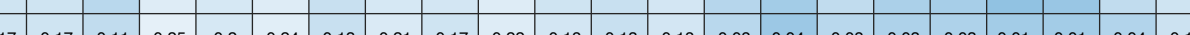

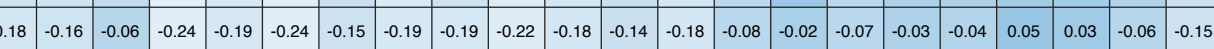

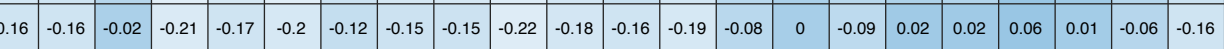
\begin{tabular}{llllllllllllll|l|l|l|l|l|l|l|l|l|l|l|}
-0.18 & -0.19 & -0.06 & -0.26 & -0.19 & -0.23 & -0.14 & -0.19 & -0.18 & -0.23 & -0.19 & -0.15 & -0.2 & -0.07 & -0.03 & -0.09 & -0.02 & 0.01 & -0.02 & -0.04 & -0.04 & -0.17
\end{tabular}

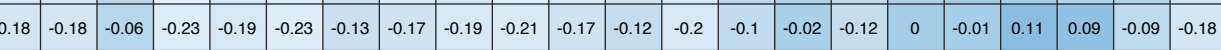

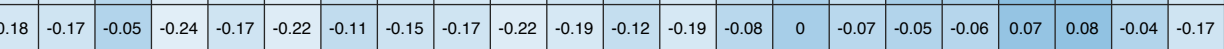
\begin{tabular}{|l|c|c|c|c|c|c|c|c|c|c|c|c|c|c|c|c|c|c|c|c|c|}
-0.19 & -0.18 & -0.04 & -0.24 & -0.18 & -0.23 & -0.15 & -0.18 & -0.16 & -0.23 & -0.19 & -0.17 & -0.19 & -0.09 & -0.02 & -0.11 & -0.07 & 0 & 0.07 & 0.02 & 0 & -0.16 \\
\hline
\end{tabular} \begin{tabular}{|l|l|l|l|l|l|l|l|l|l|l|l|l|l|l|l|l|l|l|l|l|l|}
0.04 & 0.03 & 0 & 0 & -0.01 & 0.02 & -0.01 & 0.05 & -0.04 & 0.07 & 0 & 0.01 & 0.42 & 0.03 & -0.03 & 0.11 & 0.03 & 0.01 & 0.02 & 0.15 & 0.11 & 0.12 \\
\hline
\end{tabular}

LM22 True GEPs 
Correlation: estimated GEPs vs true GEPs

\begin{tabular}{|l|l|l|l|l|l|l|l|l|l|l|l|l|l|l|l|l|l|l|l|l|l|l|}
\hline B cells naive & 0.11 & 0.1 & 0.08 & 0.14 & 0.08 & 0.13 & 0.07 & 0.17 & 0.09 & 0.17 & 0.13 & 0.11 & 0.31 & -0.01 & 0 & 0.02 & -0.02 & 0.01 & -0.03 & -0.01 & 0.11 & 0.03 \\
\hline
\end{tabular} \begin{tabular}{|l|l|l|l|l|l|l|l|l|l|l|l|l|l|l|l|l|l|l|l|l|l|l|}
\hline B cells memory & 0.13 & 0.12 & 0.04 & 0.21 & 0.2 & 0.25 & 0.08 & 0.22 & 0.14 & 0.17 & 0.11 & 0.1 & 0.08 & -0.02 & -0.02 & -0.01 & -0.03 & -0.01 & -0.03 & -0.03 & 0.07 & 0 \\
\hline
\end{tabular}

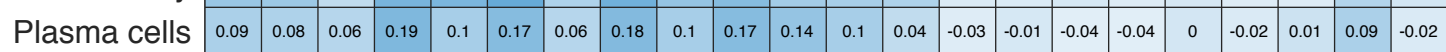

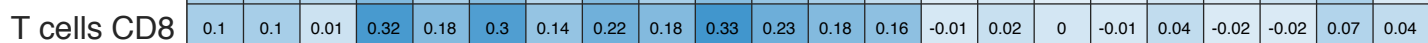

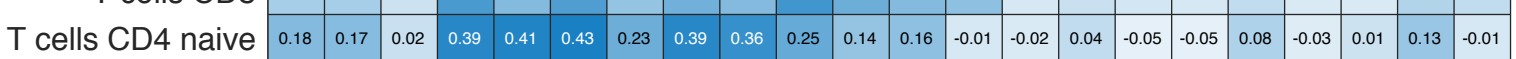

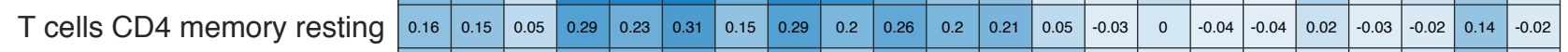

T cells CD4 memory activated

$T$ cells follicular helper

T cells regulatory (Tregs)

$T$ cells gamma delta

NK cells resting

NK cells activated

Monocytes

Macrophages M0

Macrophages M1

Macrophages M2

Dendritic cells resting

Dendritic cells activated

Mast cells resting

Mast cells activated

Eosinophils

Neutrophils

\begin{tabular}{|c|c|c|c|c|c|c|c|c|c|c|c|c|c|c|c|c|c|c|c|c|c|}
0.13 & 0.11 & 0.05 & 0.21 & 0.18 & 0.25 & 0.11 & 0.25 & 0.1 & 0.21 & 0.16 & 0.15 & 0.1 & 0 & -0.03 & -0.01 & -0.03 & -0.01 & -0.03 & -0.01 & 0.14 & 0 \\
\hline 0.18 & 0.17 & 0.09 & 0.2 & 0.26 & 0.26 & 0.09 & 0.3 & 0.22 & 0.09 & 0.03 & 0.04 & 0.13 & -0.02 & 0.01 & -0.02 & -0.03 & 0.04 & -0.03 & 0 & 0.15 & 0
\end{tabular}

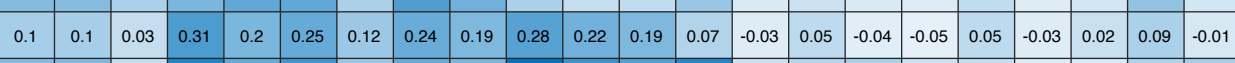

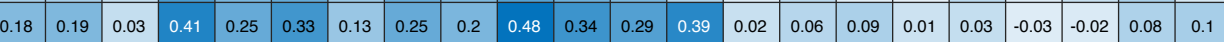

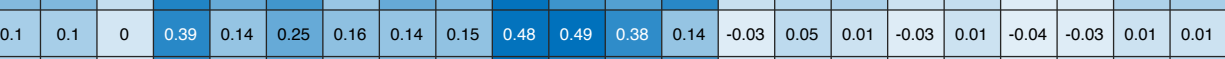

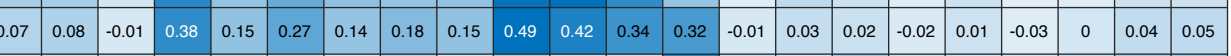

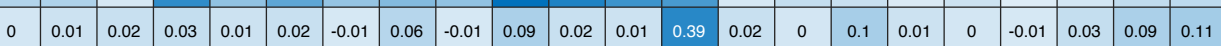

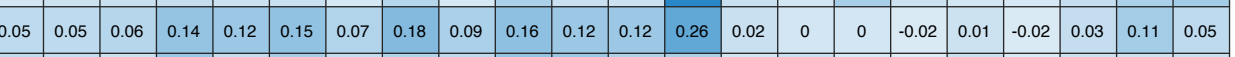

\begin{tabular}{|l|l|l|l|l|l|l|l|l|l|l|l|l|l|l|l|l|l|l|l|l|l|}
0.04 & 0.03 & 0.01 & 0.12 & 0.03 & 0.1 & 0.03 & 0.07 & 0.05 & 0.14 & 0.12 & 0.08 & 0.09 & 0 & 0.02 & -0.01 & -0.03 & 0.02 & -0.02 & 0.01 & 0.01 & 0.02 \\
\hline
\end{tabular}

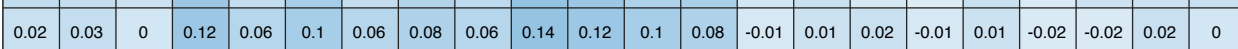

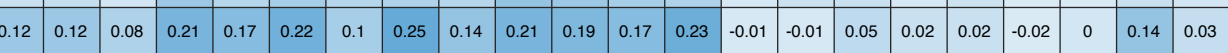

\begin{tabular}{llllllllllllllllllll|l|l|l|l|l|l|}
0.14 & 0.11 & 0.03 & 0.25 & 0.09 & 0.17 & 0.09 & 0.15 & 0.05 & 0.32 & 0.23 & 0.17 & 0.2 & -0.01 & 0.06 & 0.04 & -0.03 & 0.06 & -0.03 & 0.02 & 0.12 & 0.03 \\
\hline
\end{tabular}

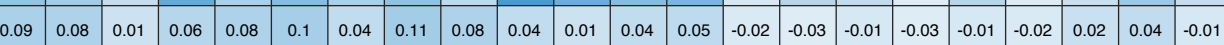

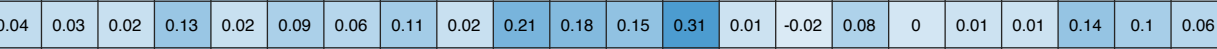

\begin{tabular}{|l|l|l|l|l|l|l|l|l|l|l|l|l|l|l|l|l|l|l|l|l|l|}
0.08 & 0.09 & 0.08 & 0.12 & 0.08 & 0.1 & 0.05 & 0.17 & 0.06 & 0.13 & 0.07 & 0.06 & 0.29 & 0.01 & 0.01 & 0.03 & -0.01 & 0.06 & -0.02 & 0.04 & 0.15 & 0.06 \\
\hline & 0.1 & 0.12 & 0.0 & 0.0 & 0.12 & 0.04 & 0.13 & 0.0 & 0.09 & 0.04 & 0.07 & 0.21 & 0 & -0.01 & 0.02 & -0.02 & 0.03 & -0.02 & 0 & 0.1 & 0.05 \\
\hline
\end{tabular}

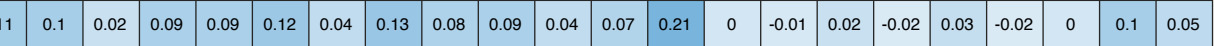

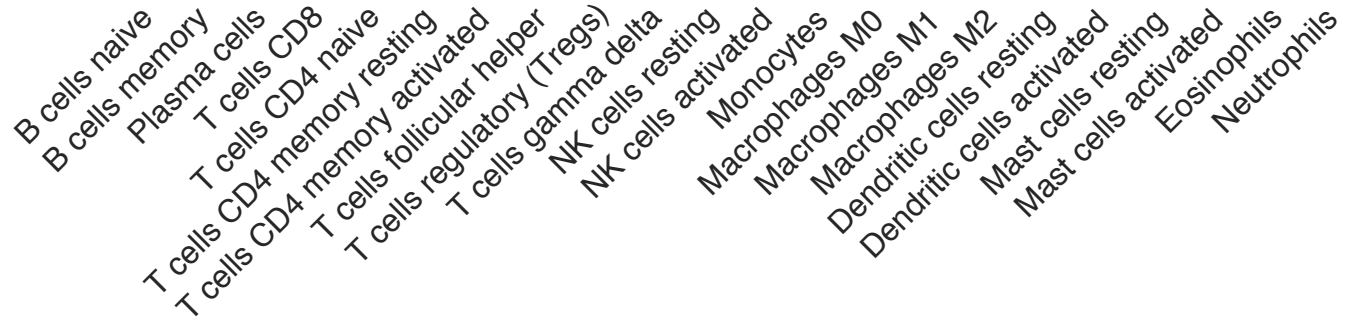

LM22 True GEPs 

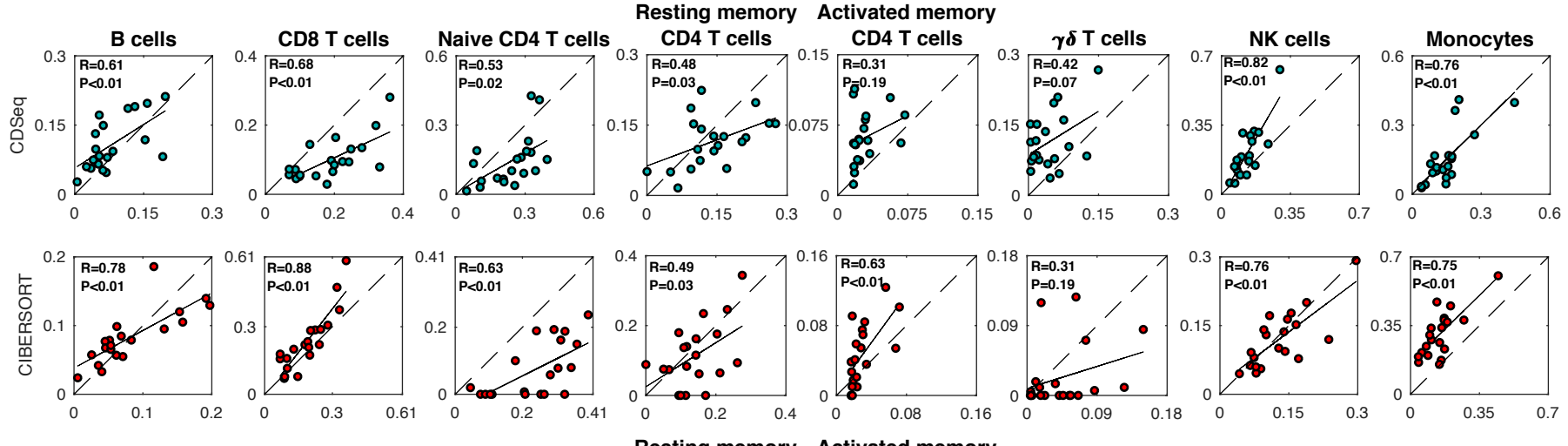

$\mathrm{H}$
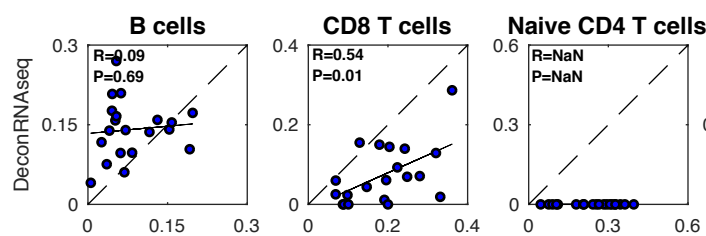

Resting memory Activated memory
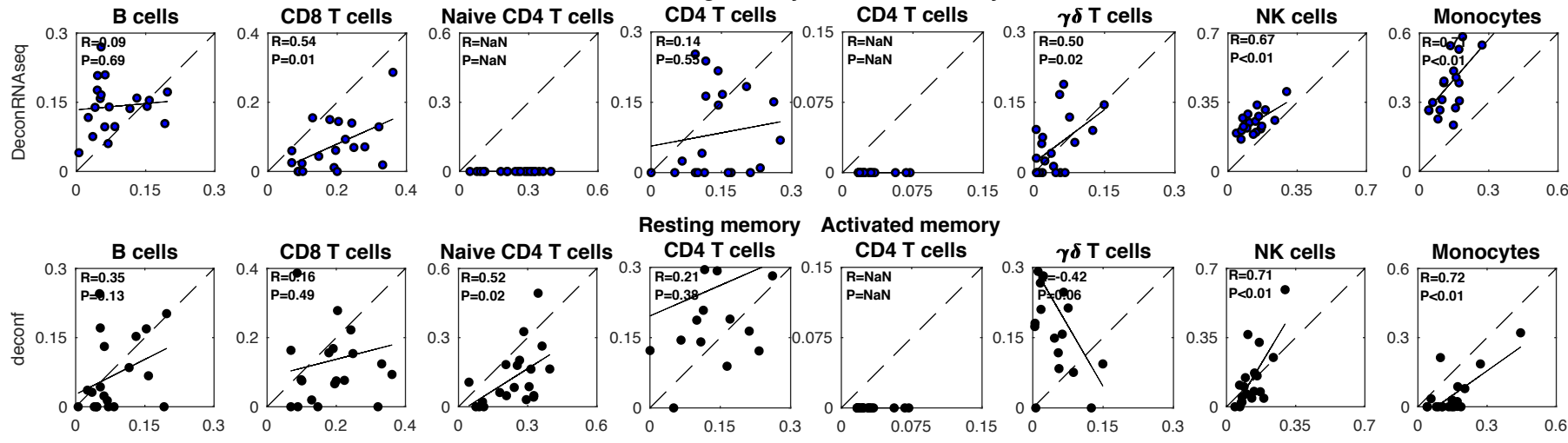

Resting memory Activated memory
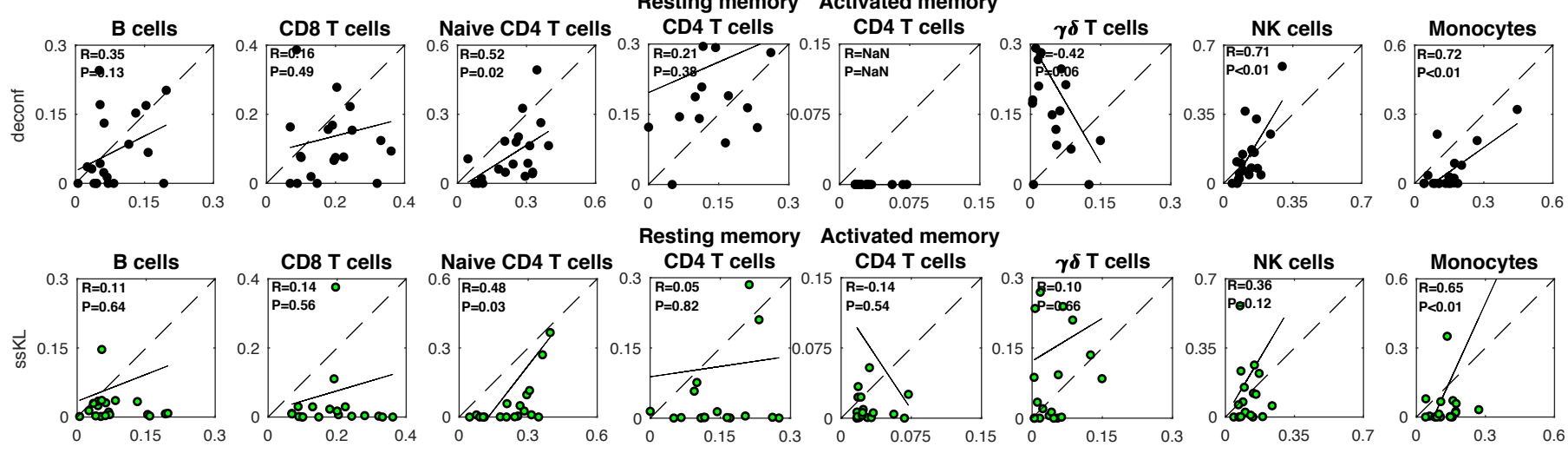


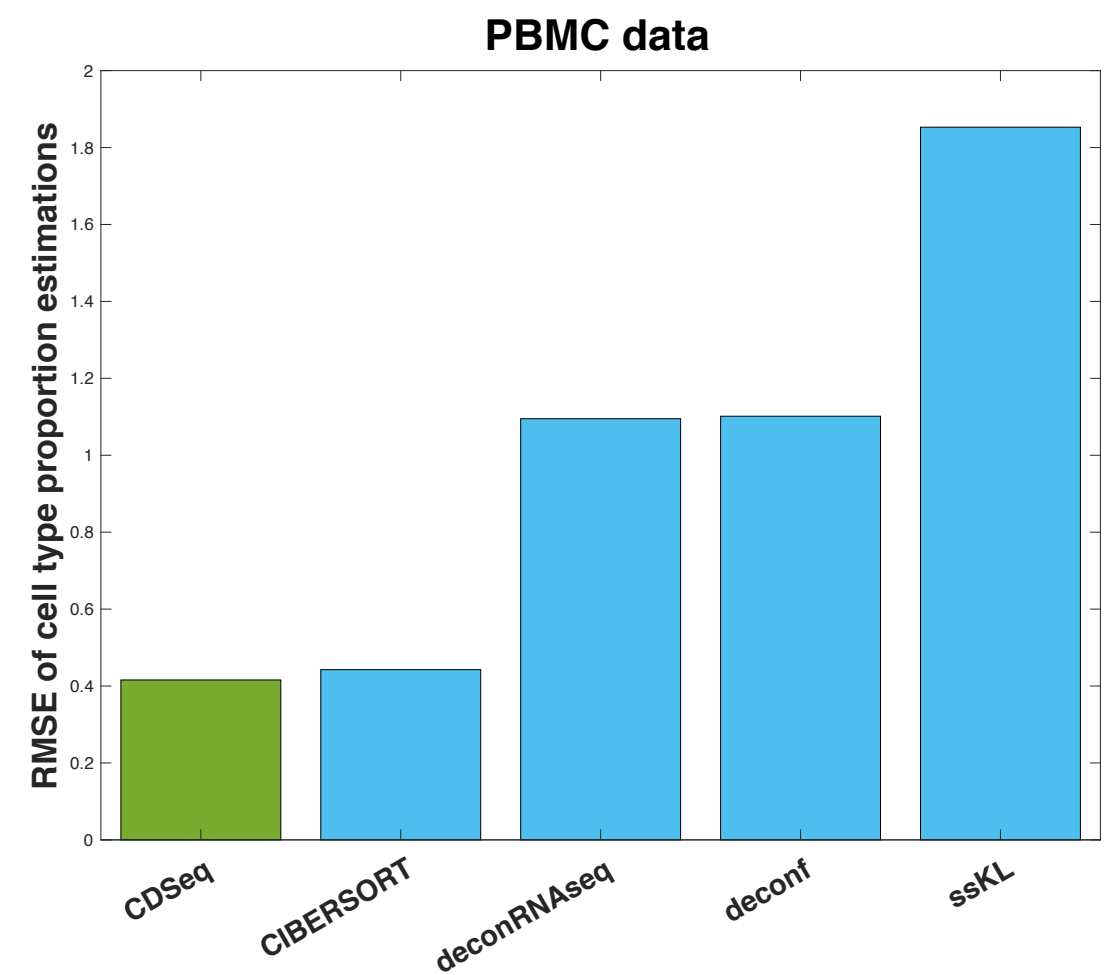

S8 Fig. Performance comparison on deep deconvolution. We applied CDSeq, CIBERSORT, DeconRNAseq, deconf, ssKL on PBMC data set. We set the number of cell types to be $22, \alpha=50, \beta=20$ for CDSeq and used default settings for other methods. $R$ is the correlation coefficient; and $P$ is the $p$-value for testing the null hypothesis of no correlation: (A) Heat map of correlations between CDSeq-estimated cell-type-specific GEPs and LM22 GEPs; and, (B) CDSeq-estimated cell-type proportions compared to flow cytometry estimates, where the upper panel (blue dots) is the result of fully unsupervised mode and the lower panel (green dots) is the result of quasi-unsupervised mode. The black line is the linear regression line; We show correlations between estimated cell-type-specific GEPs and LM22 GEPs by different methods: (C) CDSeq with quasi-unsupervised learning (heatmap of correlations of true GEPs is given in S5 Fig.); (D) deconf; (E) ssKL; We show estimated SSP versus flow-cytometry measurements by different methods: (F) CDSeq with quasiunsupervised learning; (G) CIBERSORT; (H) DeconRNAseq; (I) deconf; (J) ssKL; (K) RMSEs of cell type proportion estimations. 